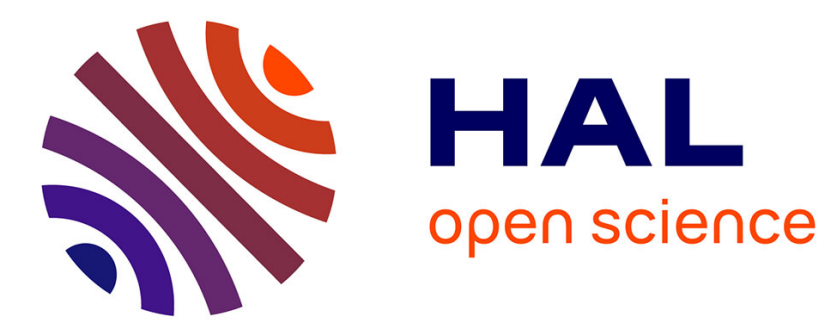

\title{
Quasistatic Plasma Sources: Physical Principles, Modelling Experiments, Application Aspects
}

K. Shamrai, Alexander Aleksandrov, G. Bougrov, V. Virko, V. Katiukha, S. Koh, E. Kralkina, G. Kirichenko, A. Rukhadze

\section{- To cite this version:}

K. Shamrai, Alexander Aleksandrov, G. Bougrov, V. Virko, V. Katiukha, et al.. Quasistatic Plasma Sources: Physical Principles, Modelling Experiments, Application Aspects. Journal de Physique IV Proceedings, 1997, 07 (C4), pp.C4-365-C4-381. 10.1051/jp4:1997430 . jpa-00255586

\section{HAL Id: jpa-00255586 https://hal.science/jpa-00255586}

Submitted on 1 Jan 1997

HAL is a multi-disciplinary open access archive for the deposit and dissemination of scientific research documents, whether they are published or not. The documents may come from teaching and research institutions in France or abroad, or from public or private research centers.
L'archive ouverte pluridisciplinaire HAL, est destinée au dépôt et à la diffusion de documents scientifiques de niveau recherche, publiés ou non, émanant des établissements d'enseignement et de recherche français ou étrangers, des laboratoires publics ou privés. 


\title{
Quasistatic Plasma Sources: Physical Principles, Modelling Experiments, Application Aspects
}

\author{
K.P. Shamrai, A.F. Aleksandrov*, G.E. Bougrov*, V.F. Virko, V.P. Katiukha, S.K. Koh**, \\ E.A. Kralkina*, G.S. Kirichenko and A.A. Rukhadze* \\ Institute for Nuclear Research, National Academy of Sciences, Kiev 252022, Ukraine \\ * Moscow State University, Moscow 117942, Russia \\ ** KIST, Cheongryang, Seoul, Korea
}

\begin{abstract}
Magnetic field enhanced rf plasma sources excited by frame-type antennas (quasistatic plasma sources) are treated theoretically and experimentally. The theoretical model predicts that a significant part of the if power is absorbed in a source plasma via the excitation of quasi-electrostatic waves. The dependences of absorption on plasma density, external magnetic field, driving frequency, and source dimensions (scaling laws) are obtained. Special experiments on low-power rf signal absorption in a preformed dense plasma corroborate well the theory. Results of test experiments with different sources have shown that a behaviour of the discharge in quasistatic sources is in good agreement with theoretical predictions. Using this knowledge, a compact low-power ion source was designed and optimized. Detailed testing of its parameters has shown that this device has good prospects for use as an ion thruster, and for various materials processing applications.
\end{abstract}

\section{INTRODUCTION}

Radio-frequency (rf) inductive methods of plasma production are used on various fields of technology from materials processing [1] to fusion devices [2,3]. These methods have many advantages for applications including a high efficiency of power utilization; a high efficiency of plasma generation, and thus a low ion production cost, and a high efficiency of gas utilization; flexibility in a source design; etc. Indeed, the density of inductively coupled plasmas grows linearly with input power [4], in contrast with capacitive discharges where the density scales as a square root of power. Plasmas with densities above $10^{12} \mathrm{~cm}^{-3}$ can be produced at a rather moderate specific input power of $50-100 \mathrm{~mW} / \mathrm{cm}^{3}$. Inductive sources are capable of running in a wide range of aspect (length-to-radius) ratios and magnetic fields, and in downstream regimes. They can also work at very low gas pressures (below $1 \mathrm{mTorr}$ ), and to produce low-energy $(<60 \mathrm{eV})$ ion fluxes onto a substrate which is favourable for highly anisotropic and damagefree processing.

Inductive sources have become the dominant tool for high aspect-ratio dry etching of sub-micron patterns [4], and for high density plasma deposition [5]. They have also good prospects for utilization as ion sources for ion thrusters [6], for gas laser pumping, plasma particle accelerators etc. [7].

Inductively coupled plasmas are known to run with or without the external magnetic field. Except for an obvious increase of plasma confinement and thus of plasma density, imposing a magnetic field improves the source operation at least in two respects [8]. First, the magnetic field increases the resistance of the plasma load, and thus the efficiency of rf power utilization and the stability of a discharge in electronegative gases. Second, it permits the discharge ignition at lower gas pressures.

From the physical viewpoint, the magnetic field gives rise to specific plasma waves, and thus changes substantially the coupling of rf power to the plasma. Excitation of waves increases the depth of $\mathrm{rf}$ field penetration into the plasma, which results in enhanced bulk power absorption.

Inductive sources can be excited by helixes, flat spirals [4], or frame-type antennas enveloping a plasma column [7,9-12]. In the latter case, the plasma production turns out to be the most efficient, probably due to a better coupling of driving antenna to waves. The usual name for devices of this type is helicon source.

In the range of plasma parameters typical for inductive sources enhanced by magnetic field, one can find in plasmas the electromagnetic (helicon) and quasi-electrostatic (Trivelpiece-Gould) waves [13-15]. Both may be excited by a frame-type antenna, but it turns out that a significant fraction of rf power is 
absorbed in helicon sources via electrostatic waves $[15,16]$. Moreover, devices of this type can operate at densities below the cut-off for helicon waves [17]. For this reason, we call a magnetic field enhanced inductively coupled plasma with a frame-type antenna as quasistatic source.

We report here the results of research on the physics and applications of quasistatic sources. Two groups of sources are treated. Low aspect-ratio (AR) sources and their applications for ground and space technologies are examined at Moscow State University. High AR sources with applications to materials processing are studied at Kiev Institute for Nuclear Research. In its physical part, this report is aimed at finding out general principles, especially scaling laws, governing source operation, and in the experimental verifying of these principles by comparing the results from sources of different design.

\section{THEORY OF A RF FIELD INTERACTION WITH A MAGNETIZED PLASMA COLUMN}

\subsection{Propagation and damping of waves in a source plasma}

In the range of plasma parameters and magnetic fields typical for the operation regimes of quasistatic sources, the following relations between the frequency of driving generator $\omega$ and characteristic plasma frequencies hold

$$
k_{z} \quad v_{\text {th }} \ll \omega_{L H}<\omega<\omega_{c} \ll \omega_{p}
$$

where $\omega_{\mathrm{p}}, \omega_{\mathrm{c}}$, and $\nu_{\mathrm{th}}$ are plasma and gyro-frequencies, and thermal velocity of electrons, respectively; $\omega_{L H}$ is the lower hybrid frequency; and $k_{z}$ is a characteristic number (along the external magnetic field $B_{0}$ ) of waves excited by a driving antenna in plasma.

Waves of two types exist in plasma in the range (1) [13]. Both belong to a magnetosonic mode but differ in wavelength. A long-wave magnetosonic mode, $k_{\mathrm{t}} c \ll \omega_{\mathrm{p}}$, is a helicon (whistler) wave. In a short-wave limit, $k_{\mathrm{t}} c>\omega_{\mathrm{p}}$, the magnetosonic mode is a quasi-electrostatic (Trivelpiece-Gould) wave. The dispersion relations for these wave are respectively

$$
\omega_{\mathrm{H}}=\omega_{\mathrm{c}} \cos \theta_{\mathrm{H}} \frac{k_{\mathrm{t}}^{2} c^{2}}{\omega_{\mathrm{p}}^{2}}, \quad \omega_{\mathrm{TG}}=\omega_{\mathrm{c}} \cos \theta_{\mathrm{TG}}
$$

where $\mathbf{k}_{\mathbf{t}}$ is a total wave-vector making an angle $\theta$ with the external magnetic field.

Helicon waves can propagate in a plasma provided that a magnetic field is not very low, so that $\omega_{\mathrm{c}}>$ $2 \omega$, and a plasma density lies in the interval which is defined by relations

$$
\omega_{\min }<\omega_{\mathrm{p}}<\omega_{\max }
$$

where

$$
\omega_{\min }=k_{z} c\left(\frac{\omega_{\mathrm{c}}}{\omega}-1\right)^{1 / 2} \text { and } \omega_{\max }=k_{z} c \frac{\omega_{\mathrm{c}}}{2 \omega^{\prime}}
$$

Electrostatic Trivelpiece-Gould (TG) waves need $\omega_{c}>\omega$, and not very high plasma density. In the range $\omega<\omega_{c}<2 \omega$, the condition for TG waves to propagate reads $\omega_{p}<\omega_{\min }$, whereas at $\omega_{c}>2 \omega$ it is $\omega_{p}<$ $\omega_{\max }$. Thus, TG waves have no the lower limit for density.

The regions where the waves can propagate are shown in Fig. 1. One can see that densities accessible for electrostatic TG waves extend far below those for helicons.

Helicon and electrostatic waves reserve the energy in different components. Almost all the energy of helicons is concentrated in a magnetic field, whereas electrostatic waves reserve it mostly in a kinetic 
energy of electrons oscillations [16]. For this reason, any electron collisions (both with neutrals and ions) are extremely effective for the electrostatic wave as destroying the principal (kinetic) part of its energy. On the contrary, any dissipation mechanism destroying either the kinetic energy of electrons (binary or anomalous collisions), or the electric field (Landau damping, or trapping of electrons by a longitudinal electric field) is ineffective for helicons. To be efficient, a mechanism of damping should destroy the magnetic field of helicon wave. Such a mechanism is absent among wave - particle interactions in plasmas of quasistatic sources.

Efficient mechanisms for the helicon dissipation are provided by wave - wave interactions, both linear and non-linear. The non-linear damping of helicon wave can arise as the beam-like parametric instability with the growth rate few-fold exceeding the driving frequency [18]. In a linear case, these mechanisms arise due to coupling of helicon wave to the electrostatic wave [15,16], that is are displayed as the mode conversion. It may occur both at the surface of a bounded plasma, or in a plasma bulk.

A bulk conversion arises if the plasma is not too dense and includes the surface $\omega_{\mathrm{p}}=\omega_{\max }$ with $\omega_{\max }$ defined in Eq. (4). At this surface, merging (degeneration) of helicon and electrostatic waves results in the efficient conversion.

If the helicon wave is excited in a bounded plasma column, it gives rise to a redundant polarization of plasma at the edge which is cancelled by arising electrostatic wave. Such a mechanism of surface mode conversion results in that a principal fraction of the energy of helicon wave is transferred to the electrostatic wave. The efficiency of this mechanism is evaluated in terms of effective collision frequency which makes $[16,19]$

$$
v_{\mathrm{eff}} \approx \omega_{\mathrm{c}}\left(r_{0} / \lambda_{z}\right)
$$

where $r_{0}$ is the plasma radius, and $\lambda_{z}=2 \pi / k_{z}$ a characteristic axial wavelength excited by antenna. Being very efficient, the surface conversion dominates in many situations as the damping mechanism for helicons,

The efficiency of surface conversion may be low provided that the azimuthal electric field of helicon

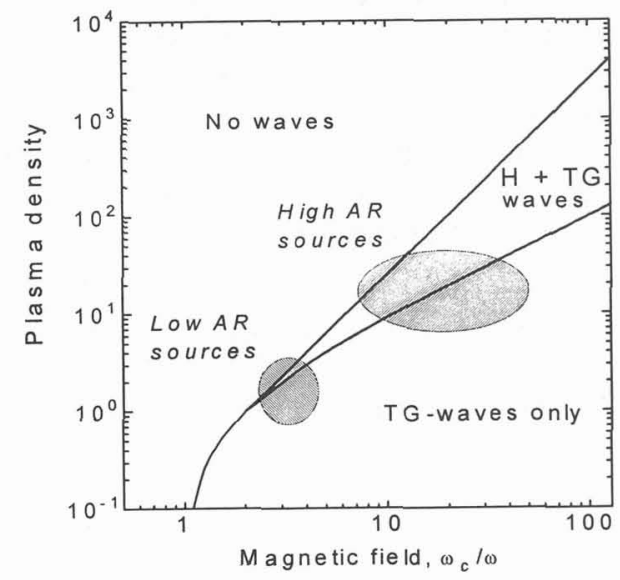

Figure 1: Regions accessible for helicon $(\mathrm{H})$ and electrostatic (TG) waves. The plasma density is in $\omega_{p}^{2} / k_{z}^{2} c^{2}$ units.

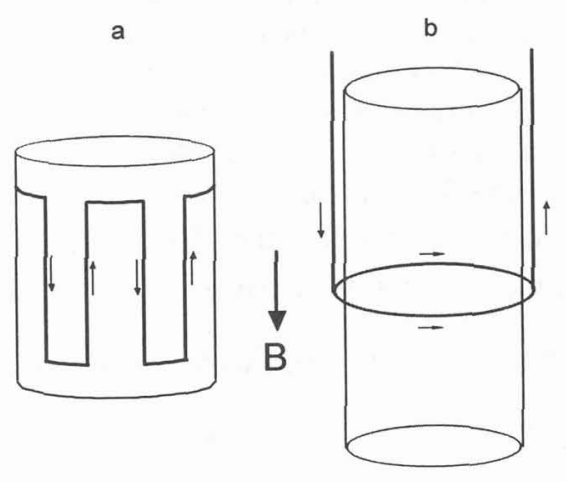

Figure 2: Antennas exciting low (a) and high (b) aspectratio sources.

wave has a node at the plasma edge. Then the redundant polarization does not arise, and the excitation of electrostatic wave is suppressed. This is the case if the plasma and wave parameters satisfy the condition of anti-resonance for the electrostatic wave excitation [20] 


$$
n=n_{\mathrm{AR}} \approx \frac{2 c}{e} \frac{B_{0}}{\omega r_{0} \lambda_{z}}
$$

Electrostatic waves excited at the plasma edge due to mode conversion propagate to the plasma centre and deposit energy via collisions. The depth of penetration is

$$
\delta=r_{0} \frac{B_{\mathrm{cr}}}{B_{0}} \quad \text { where } \quad B_{c \mathrm{r}}=\frac{m_{\mathrm{e}} c}{2 \pi e} \frac{\omega^{2}}{v} \frac{\lambda_{z}}{r_{0}}
$$

An electrostatic wave can penetrate into the bulk plasma at low magnetic fields only, $B_{0}<B_{c r}[16]$.

\subsection{Antenna-to-wave coupling and rf power absorption}

Frame-type $\mathrm{rf}$ antennas of different design were shown to be efficient for driving quasistatic sources (e.g. [7,9-12]). Two of them used in experiments are shown in Fig. 2. Both are surface current-carrying contours including axial (along the magnetic field) and azimuthal parts.

Coupling of the if driving system to the plasma may be inductive or capacitive [1]. The first is provided via rf currents whereas the second via rf charges induced in elements of the driving system. Both types of coupling are intrinsic for quasistatic sources [21]. A source can operate in a mode with low plasma density when a capacitive-type coupling is probably dominating. The plasma density in a capacitive mode is well below the cut-off for helicon waves, $\omega_{p}<\omega_{\min }$ with $\omega_{\min }$ defined by Eq. (4).

Much more interesting, however, is a high-density mode of quasistatic source which arises with strong inductive coupling. A transition between these modes occurs as abrupt density jump at smoothly varying input power $[11,22]$. In the high mode, the plasma density is normally well above the cut-off for helicons, and traveling waves may arise in the regions of plasma remote from antenna [21,23,24].

Inductive coupling is efficient provided that plasma density exceeds the cut-off value for helicons. To consider pure inductive coupling, one should put $\nabla \cdot \mathbf{j}_{\mathbf{a}}=0$, where $\mathbf{j}_{\mathbf{a}}$ is the density of antenna current. In this case, the antenna is found to be coupled to helicon waves only, and by azimuthal parts only [16]. Being excited by antenna, helicon waves transfer a principal part of energy, via surface or bulk mode conversion, to electrostatic waves which finally deposit the rf power in the plasma. Thus, helicon waves are a sort of transmission element between the antenna and electrostatic waves.

The surface mode conversion results in a bulk power absorption if the magnetic field is rather low, $B_{0}<B_{\mathrm{cr}}$, where $B_{\mathrm{cr}}$ is defined in Eq. (7). At higher fields, this mechanism gives a surface power absorption. The bulk mode conversion, if any, results normally in a bulk absorption.

With capacitive coupling $\nabla \cdot \mathbf{j}_{\mathrm{a}} \neq 0$, and antenna charges can excite electrostatic waves directly [17]. This mechanism may be efficient especially in plasmas with density well below the cut-off for helicons.

\subsection{The power absorbed with pure inductive coupling, and the discharge stability}

The rf power absorbed in the plasma is defined by the Joule law

$$
P_{\mathrm{abs}}=(1 / 2) R_{\mathrm{p}} I_{\mathrm{a}}^{2}
$$

where $R_{\mathrm{p}}$ is the plasma resistance, and $I_{a}$ is the amplitude of antenna current. The resistance is defined mainly by the excitation of waves. The model assuming that the plasma resistance is due to surface mode conversion yields the following scaling laws for the magnitude of resistance and for the location of its maxima

$$
R_{\mathrm{p}} \propto \frac{r_{0}}{\lambda_{z}^{2}} \frac{B_{0}}{n} \quad \text { and } \quad r_{0} \lambda_{z} \omega \frac{n}{B_{0}}=\text { const. }
$$


The variation with density of power absorbed in the plasma is shown in Fig. 3 for a fixed magnetic field and some value of antenna current. It is calculated for the anti-symmetric antenna shown in Fig. $2 \mathrm{~b}$, and in the approximation of pure inductive coupling described in Refs. [16,19].

One can see a non-monotonic behaviour of absorbed power in Fig. 3. The peaks of absorption arise provided that the amplitude of helicon wave is a maximum at the plasma edge, so that the efficiency of surface conversion is high. The minima are due to anti-resonances of various spatial (axial and radial) modes. The first minimum is positioned where the density satisfies condition (6).

Power losses are shown in Fig. 3 as scaling linearly with density. This is so provided that axial losses are dominant [1] which is normally true for quasistatic sources. Of two equilibrium points satisfying the power balance condition $P_{\text {abs }}=P_{\text {loss }}$, the stable one belongs to the part of absorption curve where the rate of changing of absorbed power is lower than that of losses.

Increasing the antenna current results in increased absorbed power. For this reason, the power balance can be satisfied provided that the antenna current exceeds some critical value $I_{1}$. At $I_{\mathrm{a}}=I_{1}$, a transition to inductive coupling occurs. This is shown in Fig. 4 for the absorption curve calculated at the external magnetic field $B_{1}$. The transition occurs as an abrupt jump of the plasma density to the value $n_{1}$.

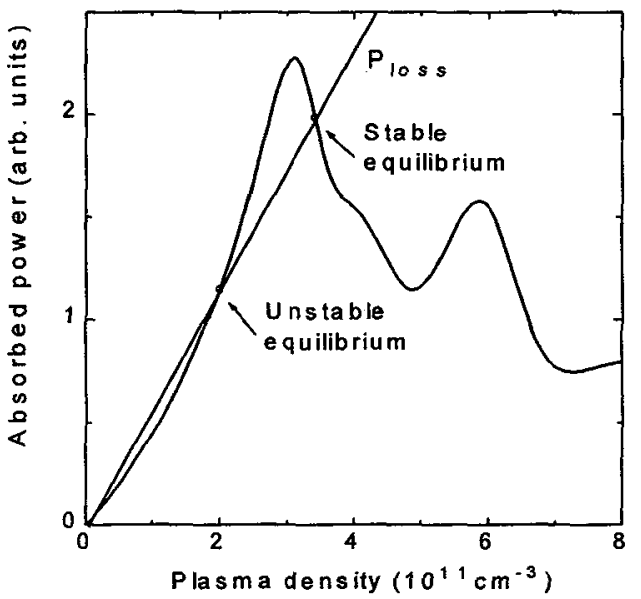

Figure 3: Stable and unstable equilibrium points for a quasistatic discharge with dominant axial losses.

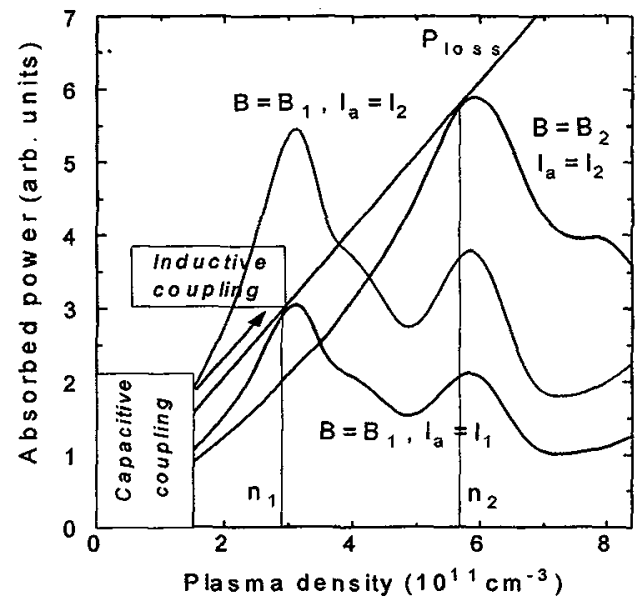

Figure 4: Transitions to inductive coupling at different magnetic fields.

Increasing the magnetic field shifts the absorption peaks to higher densities, as is seen from the second Eq. (9). As a result, at higher magnetic field, $B_{2}>B_{1}$, a transition to inductive coupling has to occur at higher antenna current, $I_{2}$, that is at higher of power. This is shown in Fig. 4 . The plasma density after this transition is also higher, $n_{2}>n_{1}$. Note that such a peculiarity of transitions was observed in a set of experiments including ours (see Sec. 3.3).

At purely inductive coupling, the absorbed power turns out to depend extremely weakly on collision frequency, as a result of a collisionless (conversion) nature of the absorption of helicon waves.

\section{MODELLING AND TEST EXPERIMENTS}

In this Section, experimental results are presented to confirm the theoretical understanding of rf power absorption in quasistatic sources, and thus to indicate the ways for finding the regimes of source operation most proper for that or another application. Experiments were conducted with various in size and design devices which are differentiated as high and low aspect-ratio (AR) devices.

Since the waves are of special importance for quasistatic sources, the most appropriate definition of the aspect ratio for these devices is as that of a characteristic wavelength excited by the antenna to the plasma radius, $\lambda_{z} / r_{0}$. Large devices like those which are used for dry etching and high-density plasma 
deposition (e.g. [22,25]) are high AR sources with $\mathrm{AR} \geq 4-5$, whereas small devices which can be used as thrusters are low $A R$ ones with $A R \approx$ 1 (see Sec. 4). In short devices, the characteristic wavelength is approximately twice a device length, $\lambda_{z} \approx 2 L$.

The regions of parameters typical for the operation modes of low and high AR sources are shown schematically in Fig. 1. High AR devices normally operate in the region accessible for helicon waves. For this reason, the power in these devices can be absorbed both via surface mode conversion, and via direct excitation of electrostatic waves arising due to capacitive coupling. The bulk conversion can work in high $\mathrm{AR}$ devices at low magnetic fields only. In low AR sources, all three mechanisms may be important, and especially direct excitation of electrostatic waves by a high-inductance antenna shown in Fig. 2a.

\subsection{Experimental study of a low-power rf signal absorption in a preformed dense- plasma column}

To refine the physical picture of $\mathrm{rf}$ field interaction with dense plasma, and to uncouple from specific discharge phenomena, we conducted experiments with a dense plasma preformed by a ECR discharge. A scheme of the experimental device is shown in Fig. 5. A quartz camera was filled by a dense plasma produced by a $2.45 \mathrm{GHz}$ ECR source which worked in a pulssed regime, $\tau_{\text {imp }}=10 \mathrm{~ms}$, at a power up to 500 W. ECR source was magnetically and microwave isolated by a magnetic screen and a wire grid. A low rf power was supplied to the antenna of the type shown in Fig. $2 b$ from a $\mathbf{r f}$ generator in the frequency range of $8-26 \mathrm{MHz}$. A variable tuning capacitor permitted matching of antennatplasma load to the generator. The plasma density was measured by a microwave interferometer.

Fig. 6 shows the interferometer trace and the variation of antenna voltage with time. One can see that the voltage varies non-monotonically, that is the plasma resistance depends non-monotonically on density. The minima of voltage correspond in Fig. 6 to maxima of plasma resistance (absorption peaks).

Fig. 6 permits one to determine the dependence of plasma resistance on density. The result is shown in Fig. 7 for different magnetic fields. Both the non-monotonic variation of resistance with density, and the location of resistance maxima, and shifting of them to higher densities with the increase of magnetic field confirm the theory of rf power absorption.

Fig. 8 demonstrates the dependence of plasma resistance at some maximum on the gas pressure. One can see that this dependence is extremely weak, as it is predicted by theory. Dropping of resistance at 


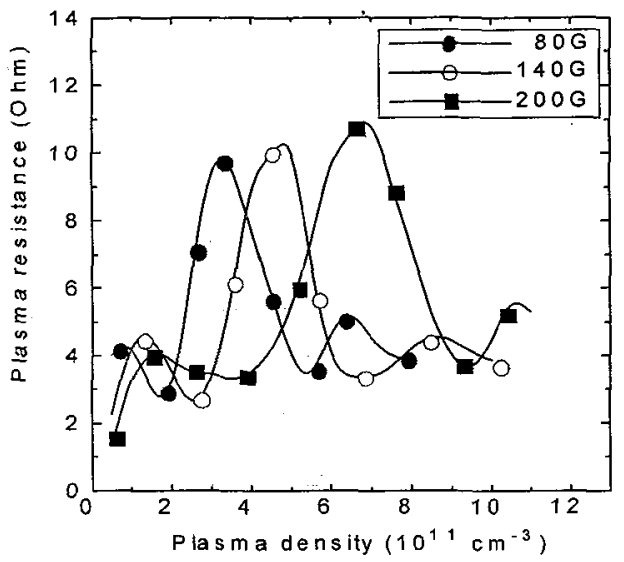

Figure 7: Resistance of a plasma load vs plasma density, at different magnetic fields.

pressures above 3 mTorr is the effect of the unstable ECR source operation at high pressures rather than some physical effect.

Relative amplitudes of different absorption peaks are shown in Fig. 9 vs axial position of the azimuthal part of antenna (double half-turn in Fig. 2b). The peaks are numbered starting from low densities (see Fig. 6). Such a behaviour is also predicted by theory supposing that each of peaks arises due to some spatial mode [16]. An exception is the first peak, its behaviour cannot be understood in the frame of purely inductive coupling. In addition, this peak lies at densities below the cut-off for helicon waves, so that it is likely the result of capacitive coupling giving rise to a direct excitation of electrostatic waves.

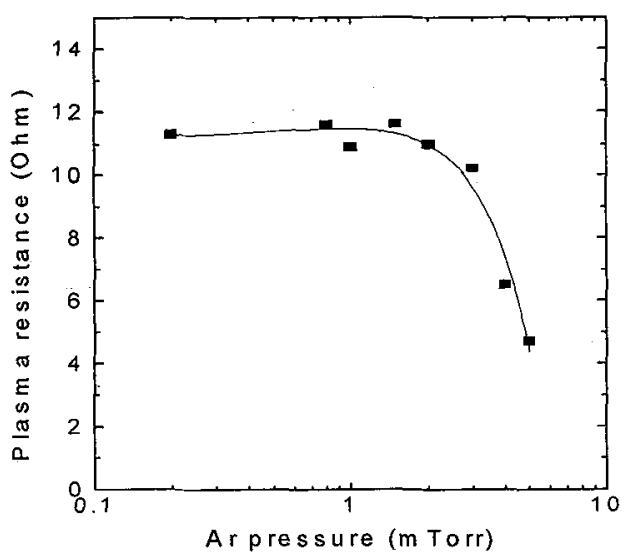

Figure 8: Dependence of a plasma resistance on $\mathrm{Ar}$ pressure.

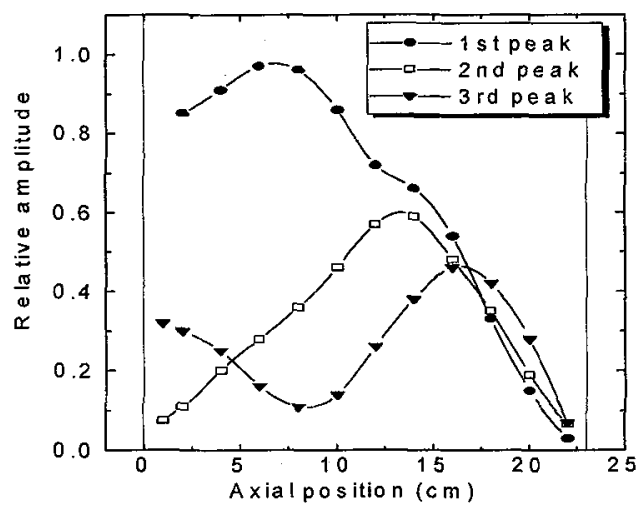

Figure 9: Amplitudes of absorption peaks as dependent on a position of antenna relative to the plasma column.

\subsection{Operation parameters of high aspect-ratio sources}

High aspect-ratio quasistatic sources are well known in applications related to materials processing where they are normally called helicon sources (e.g. [7,22,25]). A schematic diagram of a processing source operating at Kiev Institute for Nuclear Research is shown in Fig. 10 [12]. It includes a source chamber with an anti-symmetric antenna of design shown in Fig. $2 b$, and a processing chamber. Such a design is typical for processing devices (see, e. g. $[22,26]$ ).

The plasma density is shown in Fig. 11 vs magnetic field for various input powers. One can see a peak at low magnetic fields where a density of the order of $2 \times 10^{11} \mathrm{~cm}^{-3}$ is produced with a specific power input in a $50 \mathrm{~mW} / \mathrm{cm}^{3}$ range. A low- $B$ density peak was also observed in other experiments [10]. It is most probably due to the bulk mode conversion which works at low fields as the additional mechanism of power absorption (see Sec. 2.2 and Fig. 1). A possibility to get high densities at low magnetic fields is important for applications as permitting the source to run with light magnetic systems.

One can see in Fig. 12 that a high AR source is capable of operating at gas pressures over a wide range from a few tenths to a few tens of mTorr without substantial change of plasma density. This may be understood from our theory (see Sec. 2) which predicts a weak dependence of the rf absorption on collision frequency. 


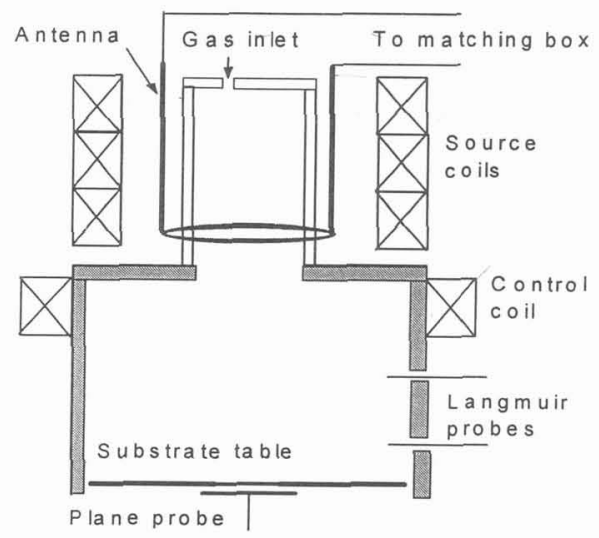

Figare 10: A scheme of a high aspect-ratio source for materials processing applications.

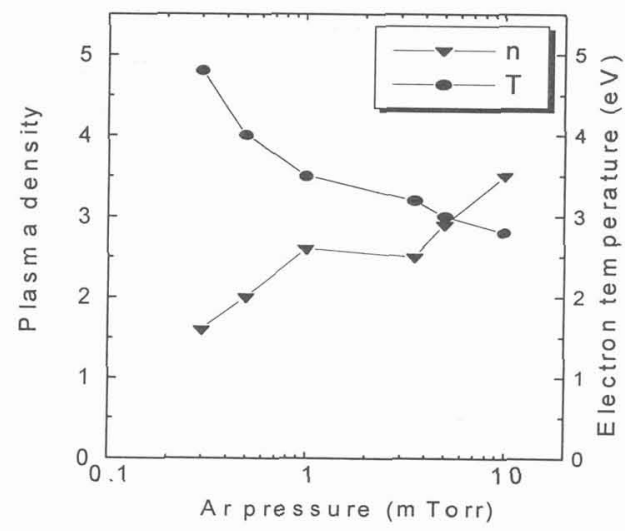

Figure 12: Plasma density (in $10^{11} \mathrm{~cm}^{-3}$ ) and electron temperature vs Ar pressure.

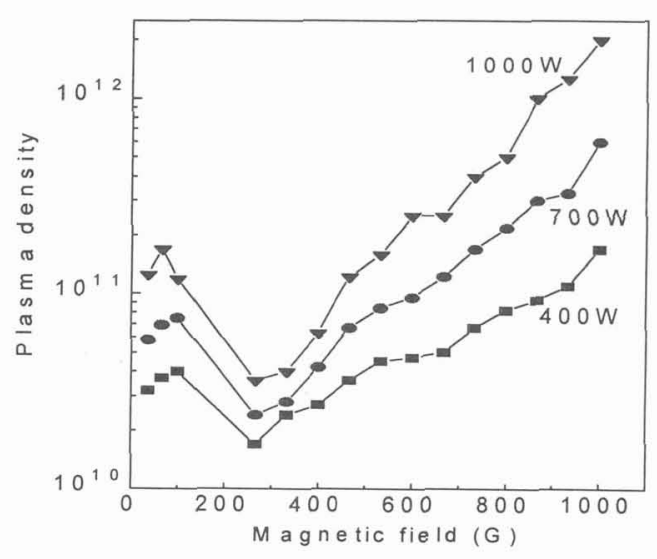

Figure 11: Variation of a plasma density with magnetic field in a high AR source, at different input powers.

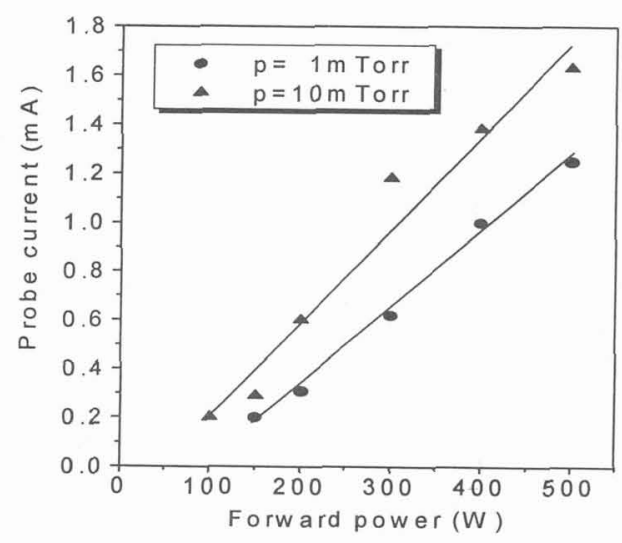

Figure 13: Ion current to probe vs forward power, at different Ar pressures.

A low sensitivity of a plasma density to the gas pressure is seen again from Fig. 13. This figure shows also a linear growth of density with input power, in agreement with the expectations of our theory. Such a growth is similar to that in a magnetic field-free source excited by a flat spiral rf coil [4].

\subsection{Optimal regimes of a low aspect-ratio low power plasma source}

Experiments with low aspect-ratio source were performed using two glass discharge tubes $9.2 \mathrm{~cm}$ in diameter and 8 and $4 \mathrm{~cm}$ long closed by end-flanges. A discharge was excited by an antenna of the type shown in Fig. 2a. It was fed by a rf generator operating at frequencies $20,30,40,60$, and $81 \mathrm{MHz}$, and at a power up to $200 \mathrm{~W}$. A magnetic field in the range of $0-600 \mathrm{G}$ was generated by a solenoid. Plasma parameters were measured by two cylindrical probes positioned at the tube axis and at a half of tube radius.

The dependences of ion saturation current on the magnetic field corresponding to the centre of the discharge tube are shown in Fig. 14 for different input powers. Dashed bands in Fig. 14 correspond to magnetic fields $\omega_{c}=\omega$, and $\omega_{c}=2 \omega$. Their finite widths are defined by a $20 \%$ non-uniformity of the magnetic field along the device.

One can see from Fig. 14 that the probe current does not change in the ECR region. However, it starts to grow rapidly at $\omega_{c}=2 \omega$, and reaches a maximum at some higher magnetic field. The increase of input 
power shifts the location of this broad maximum towards higher fields. The plasma density and its rate of growth were found to be higher at the tube periphery.

As is see from Fig. 14, the increase of the probe current and thus of plasma density starts at the magnetic field corresponding to the lower boundary for the excitation of helicon waves (see Fig. 1). In addition, a direct excitation of TG-waves is possible in the range $\omega_{c}>\omega$. We suppose this to be an evidence of efficient plasma production due to the excitation of waves in a quasistatic source.

Fig. 15 shows the variation of saturation ion current with input power at fixed values of magnetic field. One can see an abrupt increase of the current occurring at some threshold value of a rf power. This value is higher for higher magnetic fields. The further increase of power above the threshold does not result in a substantial change of ion current.

'The jumps in discharge density are likely to be the abrupt transitions from capacitive to inductive coupling we discussed in section 2.3. Similar phenomena were observed in high aspect-ratio devices $[22,25]$, and seem to be some universal property of quasistatic sources.

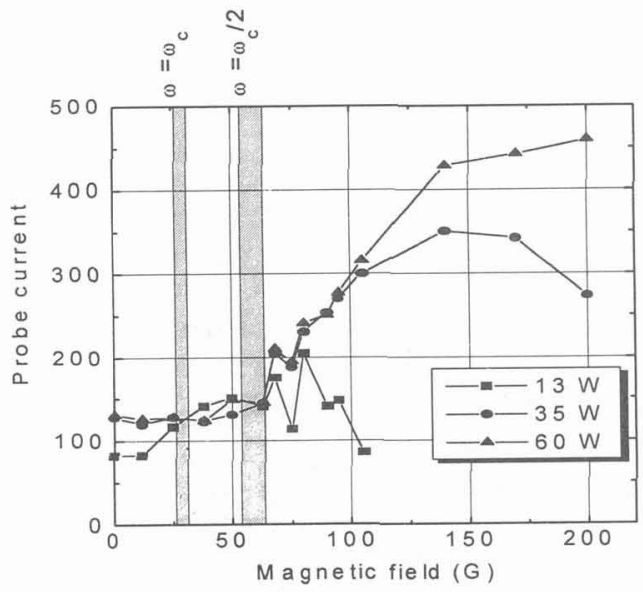

Figure 14: Dependences of saturation ion current to probe (in $\mu \mathrm{A}$ ) on magnetic field.

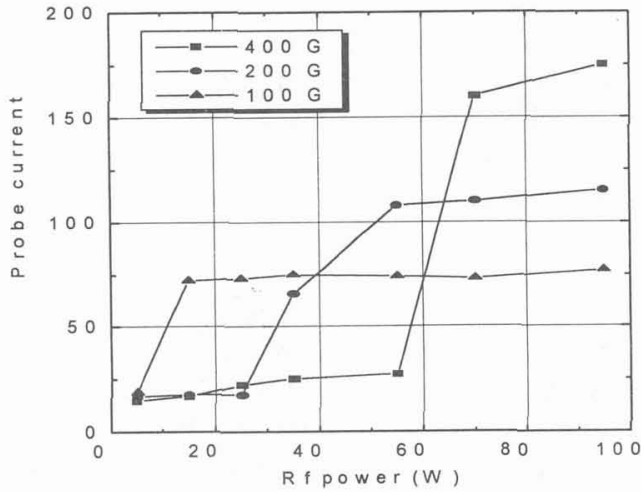

Figure 15: Dependences of saturation ion current (in $\mu \mathrm{A}$ ) on $\mathrm{rf}$ power.

\section{DESIGN AND OPTIMIZATION OF A LOW ASPECT-RATIO ION SOURCE}

The idea of low power ion source development as ion propulsion system for light communication satellites was suggested by PROEL specialists in 1991 [27]. At the same time, the development of ground plasma technologies, such as sputtering, ion assisted deposition of thin films, surface modification of materials etc.[28,29], required the new generation of low-power ion sources capable of producing pure ion beams of inert and chemically reactive gases in the range of ion current densities of $0.05-5 \mathrm{~mA} / \mathrm{cm}^{2}$, and beam energies of 100-2500 eV. These two factors initiated the start at the end of 1992 of work developing a new ion source in RIAME MAI under a partial financial support of the firm PROEL TECHNOLOGIE. The evaluation of the ion source in ground plasma technologies started in 1995 in collaboration with KIST specialists.

The comparison of ion sources has shown that dc ion sources; for instance, ion sources with divergent magnetic field [30], were very effective from the viewpoint of power utilization. They found wide application both in space technology as space ion propulsion systems and in ground industry for various ion-beam processing technologies. However, in spite of the efforts of numerous specialists, dc ion sources still have problems with the lifetime of cathodes, which decrease the period of a source's continuous work, especially with using reactive gases, pollute the ion beam, and significantly complicate source design. Complicated design is also typical for microwave ion sources. From this point of view, $\mathrm{rf}$ ion sources are much more attractive. They provide a possibility of obtaining beams of inert and chemically reactive gases with a little amount of impurities; have large resource limited by the lifetime of 
ion extraction system only, and relatively low power expenditures [28,29-31]. These advantages motivated the choice of a rf discharge for our needs, which was confirmed by that the rf ion source RITA was successfully used as an ion thruster during space mission EURECA [6]

We used the results described in Section 3.3 for designing the low-power ion source, and for choosing the region of parameters for its operation.

\subsection{Experimental installation}

Tests of a low-power $\mathrm{rf}$ ion source were performed using the installation shown in Fig. 16. The source was fastened to an adjusting flange 8 of a large vacuum chamber 3 .

We examined the ion source behaviour enhanced by magnetic field which was produced either by solenoid, or by permanent magnets. Fig. 17 shows schematically the source equipped by permanent magnets. The source with a solenoid was of similar design. The discharge chamber was a $9.2 \mathrm{~cm}$ in diam dielectric cylinder made of quartz, Pyrex, or glass ceramics, and closed at one of the ends.

The discharge was ignited by a silver or copper antenna located on the outer surface of the discharge chamber. The rf power up to $200 \mathrm{~W}$ was supplied through a matching network 13 (see Fig. 16) from a rf generator operating at frequencies $20,30,40,60$, or $81 \mathrm{MHz}$.

The ion optic system (IOS) was made as a three-electrode, three-grid system. It is non-flat and includes special "deflect" of grids and "pinched" acceleration electrode. The shape of electrode deflection is aimed at supporting the hole alignment under possible warping, minimizing the ion current density losses onto target, and achieving the necessary uniformity of the ion beam profile on the substrate. Both concave and convex grids were examined in this work.

The IOS was designed as one assembly unit. The electrodes are made of titanium alloy OT4-1. The $0.4 \mathrm{~mm}$ in width emission electrode has a transparency about 0.65 (the holes of $2 \mathrm{~mm}$ in diameter with distances of $0.5 \mathrm{~mm}$ between them). The acceleration electrode is a grid with holes of $0.8 \mathrm{~mm}$ in diameter and $0.8 \mathrm{~mm}$ in width. The transparency of this electrode is about 0.15 . The deceleration electrode is a grid with holes of $2 \mathrm{~mm}$ in diameter and $0.8 \mathrm{~mm}$ in width. The gap between the emission and the acceleration electrode is about $1 \mathrm{~mm}$; between the acceleration and the deceleration electrode 1.2 to $1.5 \mathrm{~mm}$. The insulation unit consists of cups, where 2 insulators made of Alumina are aligned using the spacers. The insulation unit serves for electrode isolation (was tested to $5000 \mathrm{~V}$ ), and carries also a mechanical load.

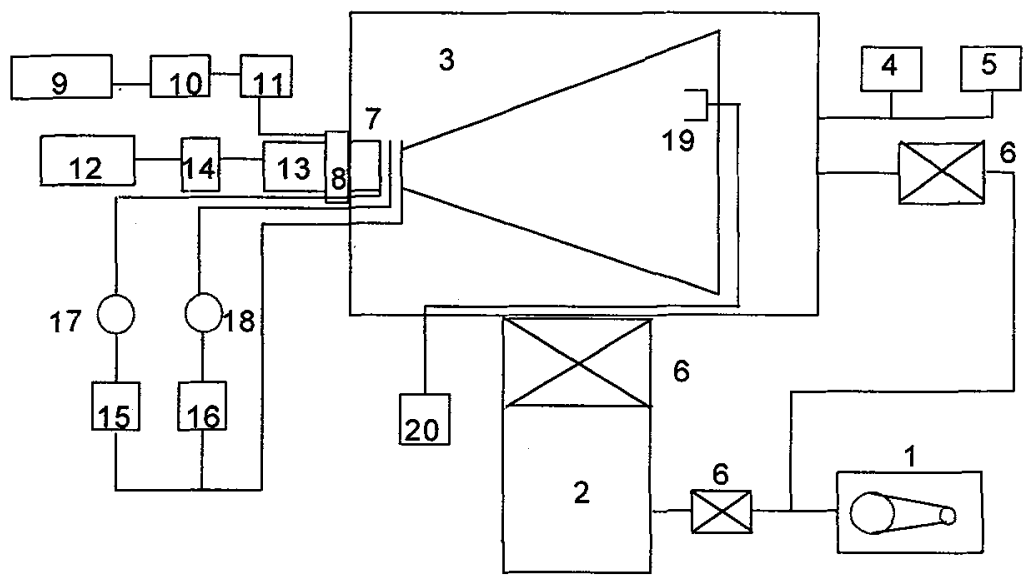

Figure 16. A scheme of experimental set-up.

1 - rotary pump; 2 - diffusion pump; 3 - vacuum chamber; 4 - thermocouple lamp; 5 -ionization lamp; 6 - vacuum seals; 7 - ion source, 8 - adjusting flange; 9 - tank with working gas; 10 - needle valve; 11 - gas flow meter; 12 - rf generator ; 13 matching box; 14 - power meter; 15, 16 - power supplies of emission and accelerating electrodes; 17, 18 - milliampermeters, 19 - Faraday cup; 20 - PC computer. 
Voltage supplies 15 and 16 (see Fig. 16) biased the emission and accelerating electrodes to potentials $0-1700$ and $0-1000 \mathrm{~V}$, respectively. The decelerating electrode was grounded. The circuit of emission electrode included a milliampermeter 17 for measuring the ion current $i_{\mathrm{b}}$ extracted from the discharge. A milliampermeter 18 measured the current $i_{\mathrm{ac}}$ from the acceleration electrode for controlling the quality of the beam optics.

To analyze the ion beam profile, a Faraday cup 19 biased to $-30 \mathrm{~V}$ was used (see Fig. 16). It could move normally to the beam axis at distances 5,22 , and $45 \mathrm{~cm}$ from the source outlet. The dependencies of ion beam density on absorbed power, gas flow rate, and acceleration potential were measured for different gases (Ar, $\mathrm{Kr}, \mathrm{Xe}$, the mixture of $\mathrm{Kr}$ with $\mathrm{H}_{2}, \mathrm{~N}_{2}, \mathrm{O}_{2}$ and air). Data were processed using $\mathrm{PC}$ computer equipped by Analog-Digital and Digital-Analog cards.

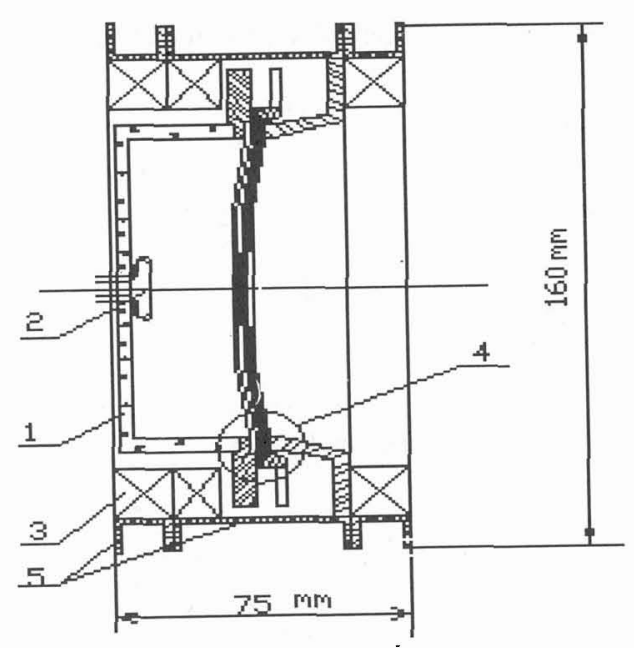

Figure 17. A schematic diagram of the rf ion source with magnetic system based on permanent magnets. 1 - discharge chamber; 2 - gas distributor; 3 - magnetic rings; 4 - ion optical system.

\subsection{Results from a source with a solenoid}

This set of experiments was performed to identify the range of magnetic fields optimal for the source operation. The source used a $8 \mathrm{~cm}$ long discharge chamber and was equipped by a solenoid producing the magnetic field up to $300 \mathrm{G}$ at the source axis. The emission and accelerating electrodes were normally biased to $+1000 \mathrm{~V}$ and $-300 \mathrm{~V}$, respectively. The experiments were conducted with a Xe consumption $m_{\mathrm{g}}$ $=0.7$ to $1.0 \mathrm{sccm}$.

Fig. 18a shows the variation of extracted ion beam current with magnetic field, at different input powers. One can see in this figure a low- $B$ peak corresponding to near-ECR region (compare with Fig. 14). At higher fields, the ion current increases without saturation.

Fig. $18 \mathrm{~b}$ shows that the coefficient of gas utilization $\beta$ (that is the ratio of extracted ion current to a gas consumption in current units, $\beta=i_{\mathrm{b}} / e m_{\mathrm{g}}$ ) behaves in a similar non-monotonic manner, and grows at rather high fields. Fig. 18c demonstrates that increasing of magnetic field above $30 \mathrm{G}$ results in the decrease of a power ion cost $C_{\mathrm{i}}$ ( a power needed for producing a unit ion current).

Figs. $18 \mathrm{~b}$ and $18 \mathrm{c}$ jointly show that at fields above $200 \mathrm{G}$ the designed device can serve as an efficient ion source. The problem with high power losses in the solenoid (about $1 \mathrm{~kW}$ ) was overcome by substituting the coil by permanent magnets.

\subsection{Optimization and tests of a source with permanent magnets}

A design of a source with permanent magnets is shown in Fig. 17. The magnetic system included magnetized rings with inner and outer diameters of 11 and $16 \mathrm{~cm}$, respectively. The rings were fabricated of a magnetically soft steel with sectors of samarium-cobalt magnets pressed in.

Increasing the magnetic field and thus the extracted current is possible in such a design by (i) using the magnetic core; (ii) decreasing the distances between rings; and (iii) increasing the number of rings. The source operation was tested with one, two, and three magnetic rings in different combinations, as well as with and without the magnetic cores. The axial magnetic field was at its largest, about $350 \mathrm{G}$, with three rings, and at its least, about $170 \mathrm{G}$, with one ring. 
The basic source model used two magnetic rings and various in length discharge chambers. The results from this model have shown that the effect of magnetic core on the beam current is low. On the
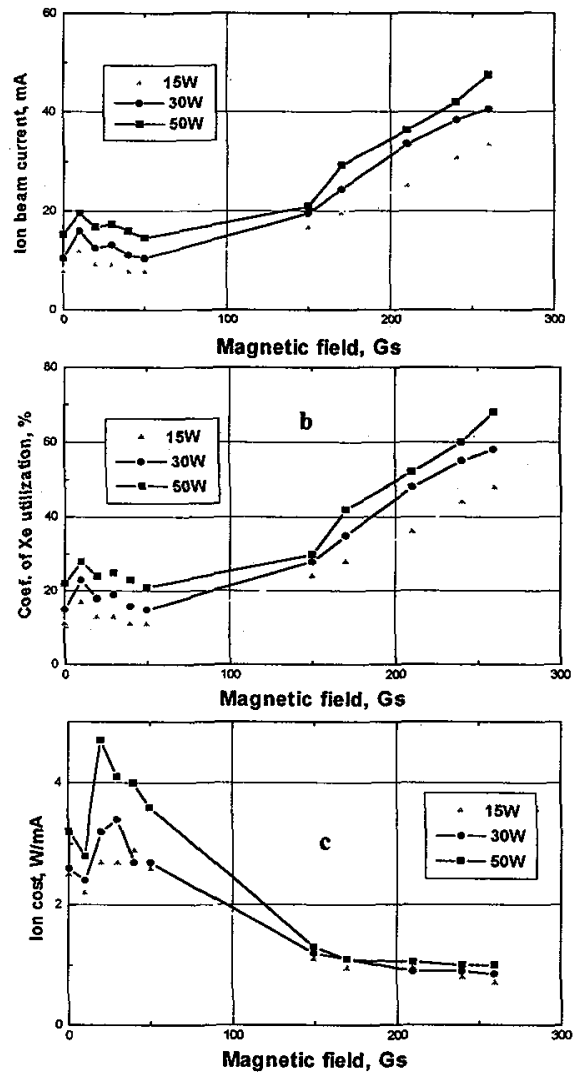

Figure 18. Dependences of Xe ion current (a), coefficient of Xe utilization (b), and ion cost (c) on magnetic field.

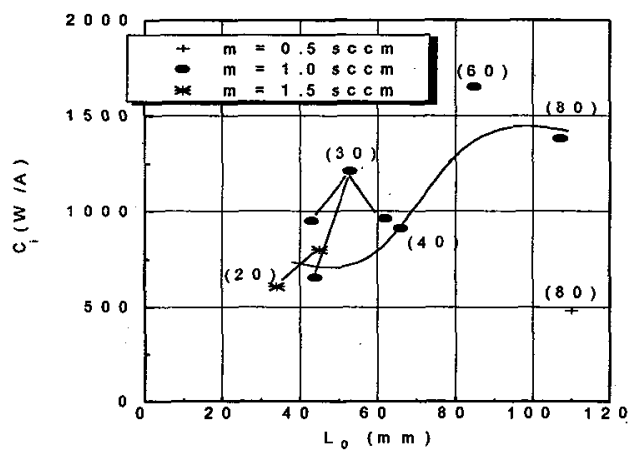

Figure 19. Dependences of $\mathrm{Xe}$ ion cost on the distance between magnetic rings for a coefficient of Xe utilization 0.7 . In brackets, the length of gas discharge chambers is indicated (in $\mathrm{mm}$ ).

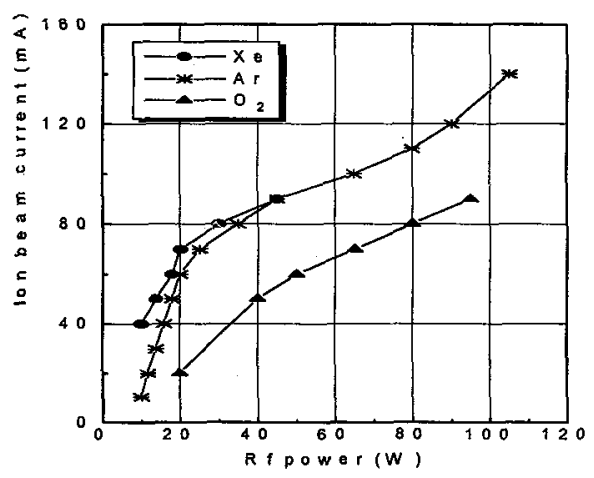

Figure 20..Dependence of ion beam current on a rf power. Magnetic system consists of three magnetic rings.

contrary, bringing the rings together and thus increasing the magnetic field gives a substantial growth of beam current.

This is seen from Fig. 19 where the ion current cost is plotted as a function of distance between the rings. Note that shorter discharge chambers were used in configurations with closer rings. Decreasing the chamber length is known to result in a decrease of power losses outside the source [32], but to reduce the lifetime of neutrals in the discharge chamber. Bearing in mind these results and those shown in Fig. 19 , we come to the conclusion that a source of optimal design should be based on a $3 \mathrm{~cm}$ long discharge chamber, and on a magnetic configuration with minimal distance between rings.

The ion current extracted from a 3-cm source was measured at a fixed input power for various gases and with different magnetic systems. Imposing a single-ring magnetic field gave a 3-fold gain in ion current, whereas adding more rings resulted in a $20-30 \%$ further increase of current only. This means that a source for technological needs may use one or two rings only. But a source for an ion thruster has to include three magnetic rings because of very strict claims to power and gas utilization efficiency.

Fig. 20 shows the variation of ion beam current with absorbed powier, for different gases. Approximately linear growth of current is seen at power above $20 \mathrm{~W}$. Such behaviour was observed in large aspect-ratio sources (see e.g. Fig. 13), and thus is an intrinsic property of quasistatic sources. The data presented in Fig. 20 for Xe are used in Sec. 5 for estimating the ion source as the ion thruster. Note that in all cases considered the current in the circuit of accelerating electrode did not exceed $1-3 \%$ of the total beam current. 
One can see from Fig. 20 the possibility of obtaining at rather low input power ion beams of inert and chemically active gases with densities in a $0.05-2.5 \mathrm{~mA}$ range. These are enough for sputtering, ion beam-assisted deposition of thin films [28,29], and other materials applications.

One more important characteristic for technological applications is beam uniformity. It is defined by three factors, that is (i) by the plasma uniformity near the IOS; (ii) by the divergence of an elementary beam extracted from a single IOS hole; and (iii) by the shape of IOS grids. Far from the source, the last two factors govern the beam profile, whereas closer to the source the first and the third factors are important.

Ion beam profiles measured close to the IOS are shown in Fig. 21. One can see a rather high uniformity, and especially for large accelerating potentials. The slight structure of the profiles is probably due to a spatial non-uniformity of fields of excited waves. The beam profile was found to be practically independent of gas, and to depend slightly on accelerating potential.
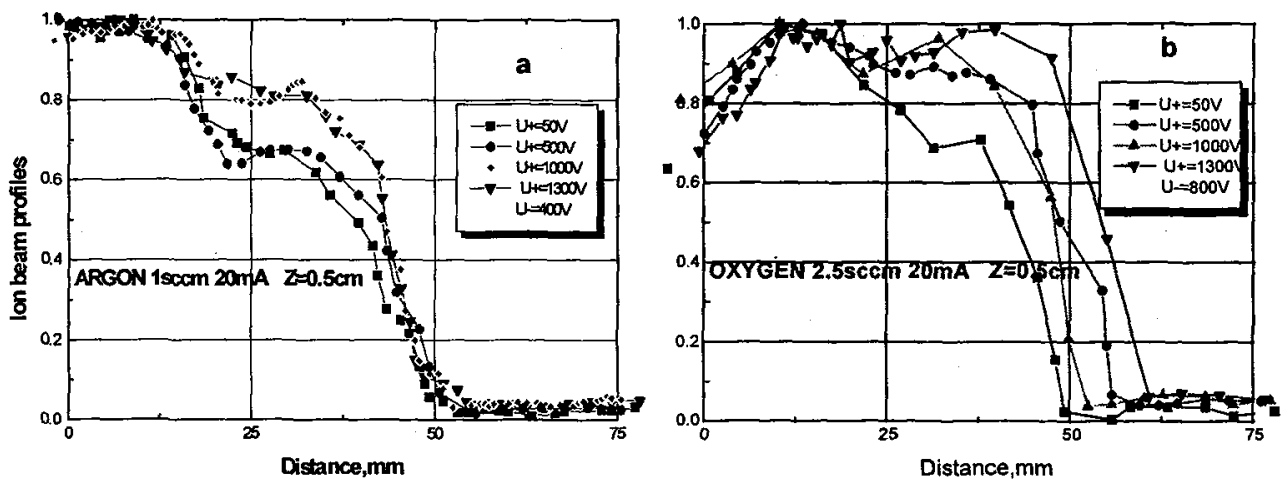

Figure 21. Ion beam profiles measured close to the exit of IOS.

The beam profiles measured at 5 and $22 \mathrm{~cm}$ distances from the source with concave IOS are shown in Fig. 22 for different magnetic configurations. The effect of magnetic configuration is of no account at $22 \mathrm{~cm}$, but plays an essential role closer to the source. This results from a divergence of elementary beams produces by each of IOS holes. The divergence of the whole beam extracted from the source with a concave IOS was measured to be within 13 to $15 \%$.

The geometry of IOS grids has a dramatic effect on the beam profile. This is seen from Fig. 23 where plotted are the profiles of beams extracted from the source with concave and convex ion optics systems.

\section{ESTIMATING QUASISTATIC SOURCES FOR APPLICATIONS}

\subsection{A prototype of an ion thruster}

To estimate a capability of the ion source with permanent magnets to serve as the ion thruster for space applications, we use the data obtained with Xe and partly presented in Fig. 20. The results are listed in Table 1 . One can see that the values of power ion cost, $C_{\mathrm{i}}=P / i_{\mathrm{b}}$, where $P$ is the input power, and $i_{\mathrm{b}}$ the

Table 1

\begin{tabular}{|c|c|c|c|c|c|}
\hline $\begin{array}{c}\text { Ion current } \\
\mathrm{mA}\end{array}$ & $\begin{array}{c}\text { Gas efficiency } \\
\%\end{array}$ & $\begin{array}{c}\text { Ion cost } \\
\text { W/mA }\end{array}$ & Specific impulse & $\begin{array}{c}\text { Thrust } \\
\mathrm{mN}\end{array}$ & $\begin{array}{c}\text { Cost of thrust } \\
\mathrm{W} / \mathrm{mN}\end{array}$ \\
\hline 60 & 70 & 670 & 3200 & 3.9 & 33 \\
\hline 70 & 70 & 500 & 3000 & 4.5 & 34 \\
\hline 100 & 70 & 700 & 3200 & 6.7 & 34 \\
\hline
\end{tabular}




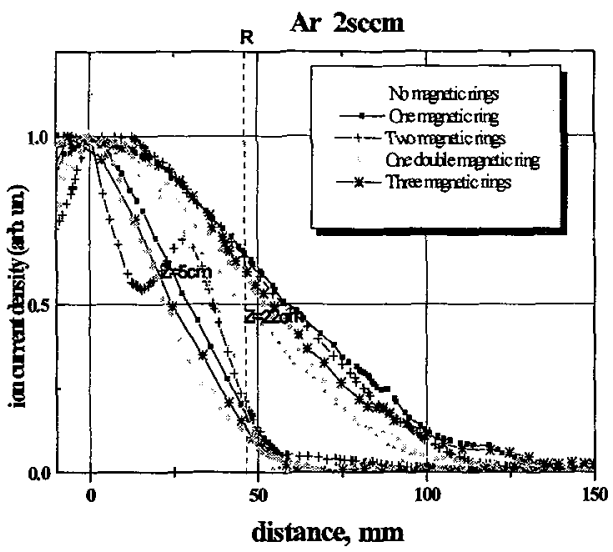

beam current, and of gas efficiency, $\beta=$ 0.7 ( $\beta=i_{\mathrm{b}} / m$ with $m$ being a gas flow rate in current units) are compatible with those obtained with RITA [6], and meet the requirements listed in [27].

Comparison of our prototype thruster with other devices can be made using Table 2 where the parameters of different low-power (the power below 1 $\mathrm{kW}$ ) ion engines are presented. All thrusters listed in this table are based on the dc discharges excepting RIT-10 which uses a rf discharge.

Figure 22. Ion beam profiles obtained with different magnetic systems and concave-type IOS.

Table 2

\begin{tabular}{|c|c|c|c|c|c|c|}
\hline lon engine & $\begin{array}{l}\text { IDOR-100 } \\
\text { USSR }\end{array}$ & $\begin{array}{c}\text { ARFA } \\
\text { Russia }\end{array}$ & $\begin{array}{l}\text { RIT-10 } \\
\text { Germany }\end{array}$ & $\begin{array}{c}\text { UK-10 } \\
\text { UK-France }\end{array}$ & $\begin{array}{l}\text { SIT-8 } \\
\text { USA }\end{array}$ & XIES \\
\hline $\begin{array}{c}\text { Thrust } \\
\mathrm{mN}\end{array}$ & 20 & 20 & 10 & 25 & 4.98 & 18 \\
\hline $\begin{array}{l}\text { Specific } \\
\text { impulse }\end{array}$ & 3300 & 3000 & 4800 & 3200 & 2600 & 2585 \\
\hline $\begin{array}{c}\text { Power } \\
\text { w }\end{array}$ & 500 & 500 & 440 & 650 & 120 & 439 \\
\hline $\begin{array}{c}\text { Ion current } \\
\mathrm{mA}\end{array}$ & 420 & 300 & 156 & & 72 & 405 \\
\hline Working gas & Cs & $\mathrm{Ar}$ & $\mathrm{Xe}$ & $\mathrm{Xe}$ & $\mathrm{Hg}$ & $\mathrm{Xe}$ \\
\hline $\begin{array}{c}\text { Gas } \\
\text { efficiency } \\
\%\end{array}$ & & & 70 & 85 & 77 & 79 \\
\hline $\begin{array}{c}\text { Power } \\
\text { efficiency } \\
\%\end{array}$ & & & 71 & & 72 & 69 \\
\hline $\begin{array}{c}\text { Total } \\
\text { efficiency } \\
\%\end{array}$ & 76 & 73 & 49 & 76 & 55 & 51 \\
\hline $\begin{array}{c}\text { Resource } \\
\text { hrs }\end{array}$ & 1,000 & 10,000 & 8,150 & 6,000 & & 12,000 \\
\hline
\end{tabular}

\subsection{A compact ion source for surface modifications of materials}

A scheme of experiments is shown in Fig. 24. Surfaces of metals and polymers were irradiated by ions of different gases accelerated to energies ranging 100 to $1500 \mathrm{eV}$. The ion beam current was controlled by the rf power and gas flow rate, and was measured by a Faraday cup biased to $-30 \mathrm{~V}$. Doses of ions in a $5 \times 10^{14}$ to $5 \times 10^{16}$ ions $/ \mathrm{cm}^{2}$ range were exposed by varying the irradiation time at fixed ion beam current. 


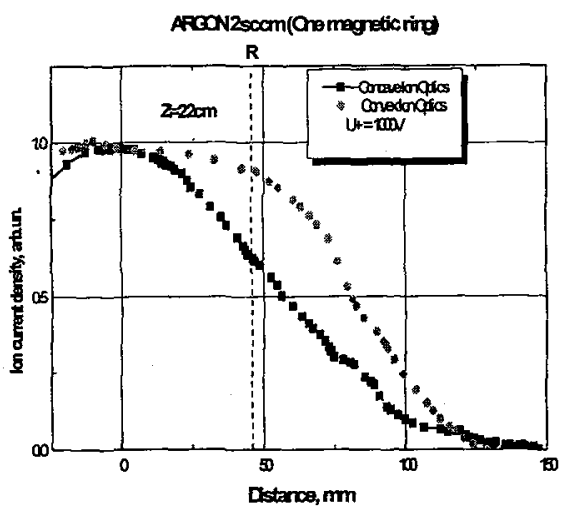

Figure 23. Ion beam profiles obtained with concave and

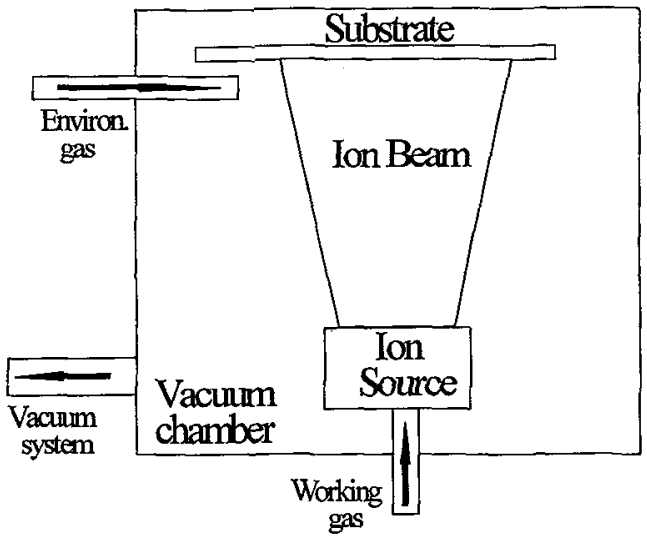

Figure 24. A diagram of the technological installation. convex grids.

Environment gas was introduced near the surface of substrate during its irradiation. A wettability of irradiated samples was measured by a contact angle meter (ERMA, Goniometer Type). The measurement was done with triply distilled water by dropping of $0.25 \mathrm{ml}$ distilled water on three different places of the substrate surface and averaging the contact angles. Polymer as well as aluminum surfaces were treated by the ion beam of different gases, with and without environment gas. Typical results of measurements for Al samples are shown in Fig. 25. One can see that the increase of ion dose up to $2 . \times 10^{17}$ ions $/ \mathrm{cm}^{2}$ leads to a significant decrease of the contact angle with hydrogen environment gas or hydrogen ions at the substrate.

The modification of Al surface was conducted using ions of inert gases and nitrogen. The modification of polymer surfaces needs also the utilization of reactive gases [33]. Fig. 26 shows the best contact angles achieved for polycarbonate in experiments with ions of both inert and chemically active gases. The results for argon and oxygen ions are close to those presented in [33,34]. The results for carbon dioxide and freon gases are new.

The operation of the ion source was stable for all gases used. The input power was less than $100 \mathrm{~W}$, and the gas flow rate did not exceed $3 \mathrm{sccm}$.

\section{CONCLUSION}

In conclusion, we designed and optimized a compact, low-power (input power below $100 \mathrm{~W}$ ) and economical (gas efficiency above 0.7 ) ion source based on a quasistatic discharge. It was shown to have a good potential as an ion thruster in a $\mathrm{mN}$ range, and as a device for surface modifications of both metals (Al) and polymers.

We established some general physical principles governing the operation of quasistatic sources, and verified their appropriateness in designing. These principles define the rf power absorption in source plasmas and permit one to predict the peculiarities of the source operation as dependent on the external magnetic field, driving frequency, and source geometry (scaling laws). Using these principles may contribute to improving the discharge stability and plasma parameters, that is to increasing the source controllability, as well as to designing new devices capable to serve as flexible instruments for various applications.

\section{Acknowledgements}

This work was partly supported by the Ministry of Science, Technologies and Industrial Policy of Ukraine (MSTIPU), Grant No 2.4/997. One of the authors (K. P. S.) acknowledges a support of his trip to the ICPIG XXIII by MSTIPU. 


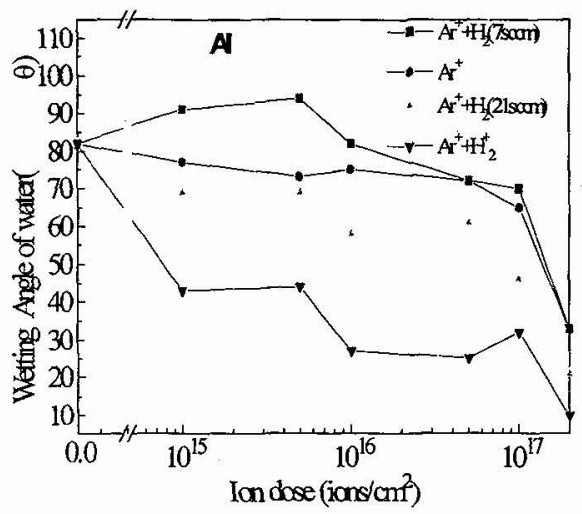

Figure 25. Change of contact angles of water for $\mathrm{Al}$ as a function of $\mathrm{Ar}^{+}$and $\mathrm{Ar}^{+}+\mathrm{H}_{2}{ }^{+}$ion dose at $1 \mathrm{keVwith}$ and without flowing $\mathrm{H}_{2}$.

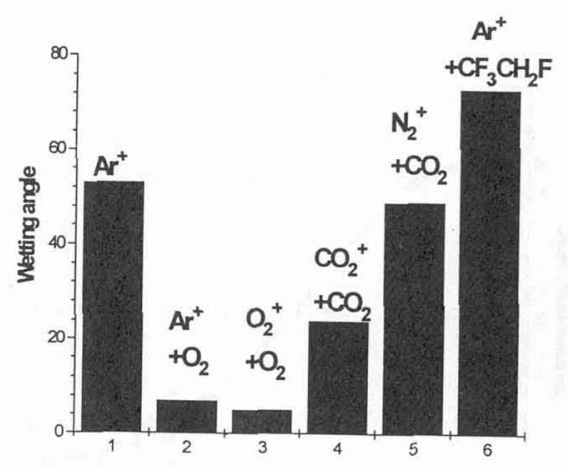

Figure 26. The best achieved contact angles for polycarbonate in experiments with ions of inert and chemically active gases.

\section{References}

[1] Lieberman M. A. and Lichtenberg A. J., Principles of Plasma Discharges and Materials Processing (Wiley, New York, 1994) pp. 387-411.

[2] Nazarov N. I., Plyusnin V. V., Ranyuk T. Yu. et al., Sov. J. Plasma Phys. 13 (1987) 871-874.

[3] Loewenhardt P. K., Blackwell B. D., and Boswell R. W., Phys Rev. Lett. 67 (1991) 2792-2794.

[4] Keller J. H., Plasma Sources Sci. Technol. 5 (1996) 166-172.

[5] Korczynski E., Solid State Technol. \# 4 (1996) 63-73.

[6] Bassner H. P., Berg H. P., et al., "Flight test results of the RITA experiment on EURECA", AIAA Papers, Sept 13-16 (1993) JEPC.

[7] Chen F. F., "Helicon plasma sources", in High Density Plasma Sources, ed. O. A. Popov (Noyes, Park Ridge, NJ, 1996) chap. 1.

[8] Keller J. H., Plasma Phys. Control. Fusion 39 (1997) A437-A442.

[9] Boswell R. W., Porteous R. K., Prytz A., Bouchoule A., and Ranson P., Phys Lett. A 91 (1982) 163164.

[10] Chen F. F. and Chevalier G., J. Vac. Sci. Technol. A 10 (1992) 1389-1401.

[11] Shoji T., Sakawa Y., Nakazawa S., Kadota K., and Sato T., Plasma Sources Sci. Technol. 2 (1993) 5-10.

[12] Katyukha V. P., Kirichenko G. S., Taranov V. B., and Shamrai K. P., Rev. Sci. Instrum. 65 (1994) 1368-1370.

[13] Aleksandrov A. F., Bogdankevich L. G., Rukhadze A. A., Principles of Plasma Electrodynamics (Springer, Heidelberg, 1984) chap. 7.

[14] Shamrai K. P. and Taranov V. B., Plasma Phys. Control. Fusion 36 (1994) 1719-1735.

[15] Vorob'ev N. F. and Rukhadze A. A., Plasma Phys. Reports 20 (1994) 1065-1077.

[16] Shamrai K. P. and Taranov V. B., Plasma Sources Sci. Technol. 5 (1996) 474-491.

[17] Aleksandrov A. F., Vorob'ev N. F., Kral'kina E. A., Obukhov V. A., and Rukhadze A. A., Zh. Tech. Fiz. 64 (1994) 53-67.

[18] Kitsenko A. B., Panchenko V. I., Stepanov K. N., and Tarasenko V. F., Nucl. Fusion 13 (1973) 557562.

[19] Shamrai K. P., Pavlenko V. P., and Taranov V. B., Plasma Phys. Control. Fusion 39 (1997) 505529.

[20] Shamrai K. P. and Taranov V. B., Phys. Lett. A 204 (1995) 139-147. 
[21] Ellingboe A. R. and Boswell R. W., Phys Plasmas 3 (1996) 2797-2804.

[22] Perry A. J., Vender D., and Boswell R. W., J. Vac. Sci. Technol. B 9 (1991) 310-317.

[23] Boswell R. W., Plasma Phys. Control. Fusion 26 (1982) 1147-1162.

[24] Chen F. F., Sudit I. D., and Light M., Plasma Sources Sci. Technol. 4 (1995) 173-181.

[25] Charles C. and Boswell R. W., J. Vac. Sci. Technol. A 13 (1995) 2067-2073.

[26] Shamrai K. P., Virko V. F., Blom H.-O., Pavlenko V. P., Taranov V. B., Jonsson L. B., Hedlund C., and Berg S., submitted for publ. in J. Vac. Sci. Technol (1997).

[27] Perrota G., Bianconi M., Bicci A., Capacci M., Cirri G. F., and Matticari G., "Orbital control and manoeuvring of lightsats / Synchronous satellites: Assessment of new ion propulsion technologies, based on electron cyclotron resonance phenomenon, to improve the performances of thrusters in the millinewton range", AIDAA/AIAA/DGLR/JSASSS 22nd Int. Electric Propulsion Conference, Viareggio, Italy, October 14-17, 1991.

[28] Rossnagel S. M., Cuomo J. J., and Westwood W. D., Handbook of plasma processing technology: fundamentals, etching, deposition, and surface interactions (Noyes, Park Ridge, NJ, 1990) 523p.

[29] Cuomo J. J., Rossnaged S. M., and Kaufman H. R., Handbook of ion beam processing technology: fundamentals, principles, deposition, film modification and synthesis (Noyes, Park Ridge, NJ, 1989), p.362.

[30] Kaufman H. R., J. Vac. Sci. Technol., 21 (1982) 725-731.

[31] Eberius M., et al., "Electric propulsion engines and their technical applications", Proc. 2nd GermanRussian Conference, Moscow, Russia, July 16-21, 1993.

[32] Alexandrov A. F., Kondranin S. G., Bougrov G. E, Vorobjev N. F., Kralkina E. A., Obukhov V. A., and Rukhadze A. A., Appl. Phys. 1 (1995) 3-25.

[33] Koh S. K., Choi W. K., Cho J. S., Song S. K., Kim Y. M., Jung H. J., J. Mater. Res. 11 (1996) 2933-2943.

[34] Choi W. K., Koh S. K., and Jung H. J., J. Vac. Sci. Technol. A 14 (1996) 2366-2378. 